\title{
Modelling the Synchrotron Emission of AGN with Grid-Based Hydrodynamic Simulations
}

\author{
Izak P. van der Westhuizen*, Brian van Soelen, Petrus J. Meintjes \\ Department of Physics, University of the Free State, 9300, Bloemfontein, South Africa \\ E-mail: VanDerWesthuizenIP@ufs.ac.za, vansoelenb@ufs.ac.za, \\ meintjpj@ufs.ac.za
}

\begin{abstract}
The spectral energy distributions of AGN have a characteristic double-hump structure, with emission at the lower wavelengths being dominated by synchrotron emission from non-thermal electrons in a relativistic jet. In order to investigate possible causes of variability in these systems we model the synchrotron emission from relativistic jets using a 3D hydrodynamic simulation. The simulation was run using the grid based hydrodynamic code PLUTO. The model setup for the simulation consisted of a uniform background medium with less dense jet material injected, at a constant rate, with a Lorentz factor of $\Gamma=10$. Post-processing calculations were applied to the simulation in order to produce $2 \mathrm{D}$ intensity maps at $15 \mathrm{GHz}$. For this emission we assumed that all of the jet particles had a power-law distribution with no cooling effects. A delta-approximation model was setup to calculate the synchrotron emission and absorption coefficients, and these were then integrated along a user defined line of sight to produce intensity maps. The simulation was evolved until just before the working surface of the jet left the computational domain. The intensity plots show the formation of multiple emission regions at the head of the jet along with a varying intensity.
\end{abstract}

4th Annual Conference on High Energy Astrophysics in Southern Africa

25-27 August, 2016

Cape Town, South Africa

\footnotetext{
* Speaker.
} 


\section{Introduction}

Active galaxies, make up the majority of objects detected in the gamma-ray sky by FermiLAT [1]. The general properties of these sources can be explained by a unified model that is very dependent on the viewing angle at which the sources are observed. The unified model of active galactic nuclei (AGN) [2] consists of a central super massive black hole (SMBH), surrounded by an accretion disc which powers the emission, beyond which it is surrounded by a dusty torus. The gas which is ionized by the thermal emission from the accretion disc, produces observed broad and narrow emission lines. Sources which are radio loud also produce strong jets, responsible for the non-thermal multi-wavelength emission from radio to $\mathrm{TeV}$ gamma-ray energies. Depending on the observing angle of the source, different properties are measured. For example radio loud systems observed close to edge-on are seen as radio galaxies, while for systems where the jet is propagating close to our line of sight, they are observed as blazars. The emission we receive from these objects are dependent on the presence and morphology of a relativistic jet and the angle of observation.

The relativistic jets observed from AGN are highly collimated, and can produce emission over $\mathrm{kpc}$ to Mpc scales. In the one-zone leptonic model, the non-thermal emission produced from the sources is the result of the acceleration of leptons in the jet in single regions which would cool via synchrotron emission and inverse Compton scattering. The spectral energy distributions (SEDs) of blazars typically exhibit a double-hump structure, showing a lower (radio to UV/X-ray) and higher (X-ray to gamma-ray) energy component, which can be modelled by these processes (see e.g. $[3,4])$. The emission is also highly variable, showing flaring events over all wavelength regimes. However, if the emission was only produced by a single population of electrons this would imply, in the simplest case, that a correlation should exist between the different wavelength regimes. While such correlations are often observed (e.g. [5, 6]), at other times sources show anti-correlations or flares only at one wavelength (e.g. [7]). This suggests either that there could be multiple zones of emission or that hadronic processes must also be included to explain the higher energy emission (e.g. [8]).

In order to investigate the possible correlation between the lower and higher energy emission in AGN jets we are using relativistic hydrodynamic simulations to model the flow of jets and use these simulations to model the non-thermal emission produced. As a first step we are using the hydrodynamic simulations to model the synchrotron emission from the jet. The hydrodynamic simulations are performed using the PLUTO package [9] while the synchrotron emission is calculated using our post-processing code.

These proceedings are structured as follows: in section 2 we briefly summarize the fluid dynamic equations, section 3 describes the setup parameters of the simulation, section 4 summarizes how the synchrotron emission is modelled, the results are presented in section 5 and the final discussion and conclusions in section 6 .

\section{Fluid dynamics}

The flow of the relativistic jet is modelled using relativistic hydrodynamics, under the assumption that on the largest scales this can accurately simulate the bulk motion of the fluid. Within the PLUTO grid-based code the fluid equations are solved and evolved with time following the 


\begin{tabular}{ll}
\hline Parameter & Value \\
\hline Lorentz factor $(\Gamma)$ & 10 \\
Jet density $\left(\rho_{j}\right)$ & $10^{-1}$ \\
Ambient density $\left(\rho_{a}\right)$ & $10^{4}$ \\
Pressure $(P)$ & $10^{-3}$ \\
Mach number & 7.8 \\
Adiabatic index $\left(\gamma_{b}\right)$ & $5 / 3$ \\
\hline
\end{tabular}

Table 1: Parameters used in the computational setup of the relativistic jet.

conservation equation [9],

$$
\frac{\partial \boldsymbol{U}}{\partial t}=-\nabla \cdot \mathbf{T}(\boldsymbol{U})+\boldsymbol{S}(\boldsymbol{U})
$$

If the co-moving density is given by $\rho$, the pressure by $P$, the enthalpy by $h$, and the Lorentz factor of the fluid is $\Gamma=\left(\sqrt{1-|\boldsymbol{v}|^{2}}\right)^{-1 / 2}$, (where $\boldsymbol{v}$ is the velocity of the fluid in units of the speed of light), then for relativistic hydrodynamics the conserved variables are given by

$$
\boldsymbol{U}=\left[\begin{array}{c}
D \\
\boldsymbol{m} \\
E
\end{array}\right],
$$

where $D=\Gamma \rho, \boldsymbol{m}=\rho h \Gamma^{2} \boldsymbol{v}$, and $E=\rho h \Gamma^{2}-P$. The tensor containing the flux terms is given by

$$
\mathbf{T}(\boldsymbol{U})=\left[\begin{array}{c}
D \boldsymbol{v} \\
\boldsymbol{m} \boldsymbol{v}+P \mathbf{I} \\
\boldsymbol{m}
\end{array}\right]^{T}
$$

where I is the unit tensor. The final term, $\boldsymbol{S}(\boldsymbol{U})$ contains any sources terms (e.g. gravity); in our simulation we do not include any source terms so in this case $\boldsymbol{S}(\boldsymbol{U})=0$.

\section{Computational setup}

The computational calculations were performed on a three dimensional computational grid of size $64 \times 64 \times 128$ unit lengths (with a computational resolution of 4 points per unit length). All calculations were performed using arbitrary units to avoid computational errors, except for velocity which is given as a fraction of the speed of light.

The simulation was initialized with a uniform stationary medium, and the jet was injected at the centre of the base of the simulation, at $(32,32,0)$, at a constant rate with a Lorentz factor of 10 , through a nozzle of radius 1 . The density ratio between the jet and ambient medium was $\eta=10^{-5}$ and a pressure match jet was used. The simulation parameters are summarized in table. 1.

The simulation was evolved with time using the HLLC Riemann solver [10] with piecewise parabolic interpolation between cells (see e.g. [11] for a general description of Riemann solvers for fluid dynamics). The simulation was evolved until the working surface of the shock left the computational domain. 


\section{Synchrotron emission modelling}

The synchrotron emission from the relativistic hydrodynamic simulation has been estimated using a post-processing PYTHON code which has been implemented to calculate the emission and absorption coefficients in each cell. The projected 2D image for any viewing angle of the simulation is calculated by integrating over defined lines of sight. Currently, the relativistic effects due to the Doppler boosting of the emission as well as the correction between the emitted and received frequencies are correctly taken into account, while the effects due to light travel time as well as correction due to cosmological redshift have not yet been implemented. However, this is sufficient to investigate where the regions of highest emission levels are occuring and how this develops with time. However, light travel effects may alter the morphology of the observed emission as shown by e.g. [12].

In order to determine the synchrotron radiation, the emission and absorption coefficients must be calculated in each cell and this requires a numerical integration over the particle energy. However, to perform this full calculation is very computationally expensive as the coefficients must be calculated $524288(64 \times 64 \times 128)$ times for every time step. Therefore, we have calculate the coefficients in the emitted frame using a $\delta$ approximation [4],

$$
j_{v}^{\mathrm{em}}=\frac{4}{9}\left(\frac{e^{2}}{m_{\mathrm{e}} c^{2}}\right)^{2} u_{B} v^{1 / 2} v_{0}^{-3 / 2} n_{\mathrm{e}}\left(\sqrt{\frac{v}{v_{0}}}\right),
$$

and

$$
\alpha_{v}^{\mathrm{em}}=\frac{2}{9} \frac{p+2}{m_{\mathrm{e}}}\left(\frac{e^{2}}{m_{\mathrm{e}} c^{2}}\right)^{2} u_{B} v^{-2} v_{0}^{-1} n_{\mathrm{e}}\left(\sqrt{\frac{v}{v_{0}}}\right),
$$

for the emission and absorption coefficients, respectively. Here, $e$ and $m_{\mathrm{e}}$ are the charge and mass of an electron, respectively, $c$ is the speed of light, $u_{B}=B^{2} / 8 \pi$ is the magnetic energy density for a field $B, v$ is frequency and

$$
v_{0}=\frac{3 e B}{4 \pi m_{\mathrm{e}} c} .
$$

Since the relativistic hydrodynamic simulation does not include a magnetic field, the energy density is calculated as a fraction of the internal energy density. The spectrum of the emitting particles is assumed to follow a power-law of the form,

$$
n_{\mathrm{e}}(\gamma)=n_{0} \gamma^{-p}
$$

where $\gamma$ is the Lorentz factor of the particles. For this first approximation we have assumed that the particles have an index of $p=1.8$ and determine the normalization, following [13], as

$$
n_{0}=\left[\frac{\mathscr{U}(p-2)}{1-C_{E}^{2-p}}\right]^{p-1}\left[\frac{1-C_{E}^{1-p}}{\mathscr{N}(p-1)}\right]^{p-2},
$$

where $C_{E}$ is the ratio of the maximum to minimum energy of the electrons, $\mathscr{U}$ is the electron energy density (approximated by a fraction of the internal energy) and $\mathscr{N}$ is the electron number density (approximated by $\mathscr{N}=\rho / m_{\mathrm{p}}$ where $m_{\mathrm{p}}$ is the proton mass). The internal energy and proper density is calculated from the numerical simulation, and we assume $C_{E}=10^{3}$. 


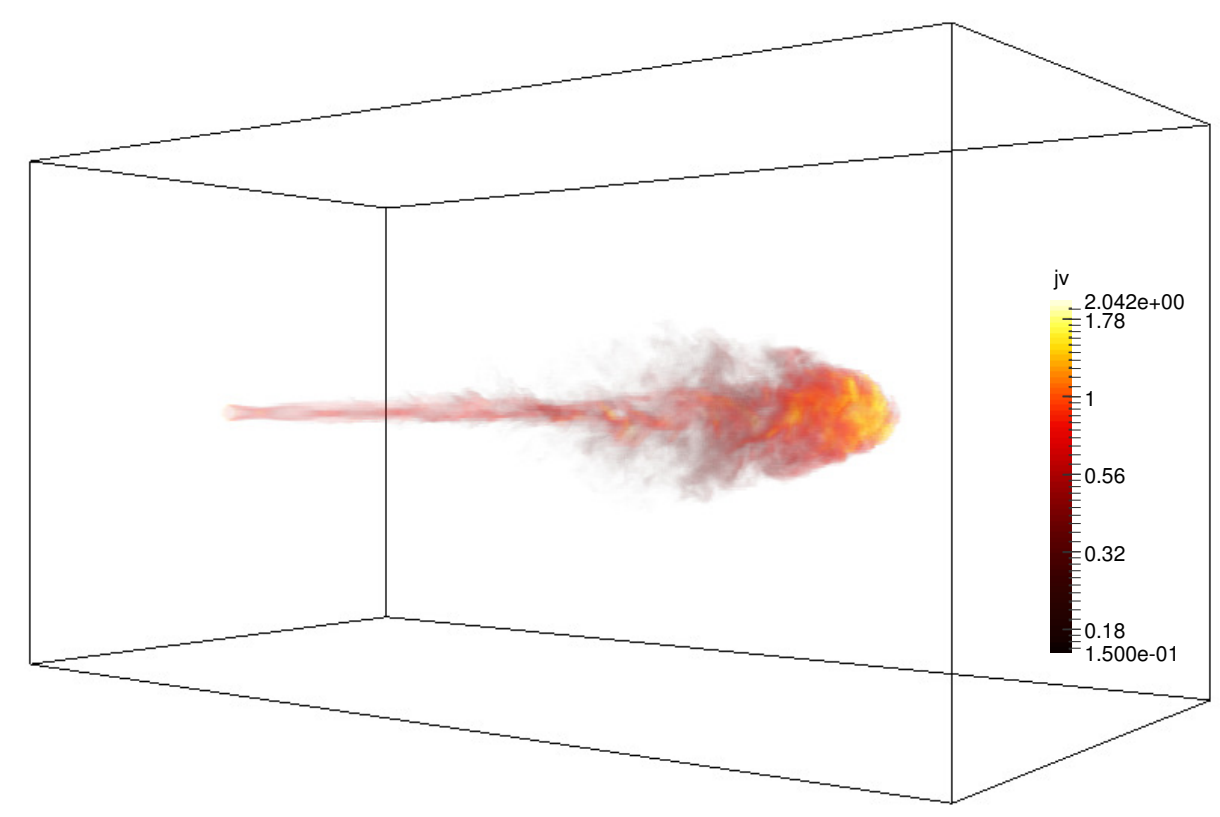

Figure 1: Frame of the three dimension simulation scaled by the co-moving emission coefficient. Please note that the units are arbitrary.

The coefficients are transformed to the observer frame by

$$
j_{v^{\mathrm{rec}}}^{\mathrm{rec}}=\delta^{2} j_{v}^{\mathrm{em}}
$$

and

$$
\alpha_{v^{\mathrm{rec}}}^{\mathrm{rec}}=\alpha_{v}^{\mathrm{em}} / \delta
$$

with

$$
\delta=\frac{1}{\Gamma(1-\beta \mu)}
$$

where $\mu$ is the cosine between the direction of motion of the fluid element and the observer, and $\beta$ is the speed in units of the speed of light. The change in intensity across a length $\mathrm{d} s$ is given by

$$
\frac{\mathrm{d} I_{v^{\mathrm{rec}}}^{\mathrm{rec}}}{\mathrm{d} s}=j_{v^{\mathrm{rec}}}^{\mathrm{rec}}-\alpha_{v^{\mathrm{rec}}}^{\mathrm{rec}} I_{v^{\mathrm{rec}}}^{\mathrm{rec}}
$$

and this is integrated along a user chosen direction to create a two dimension projection.

\section{Results}

Figure 1 shows a snap shot of the 3D simulation, scaled by the co-moving emission coefficient. This shows the morphology of the jet and the dominance of the emission near the working surface.

Figure 2 shows the $2 \mathrm{D}$ projections of the emission from the jet simulation at six equal time steps. This has been calculated for a received frequency of $15 \mathrm{GHz}$ and for a viewing angle which is perpendicular to the direction of the injected jet. 

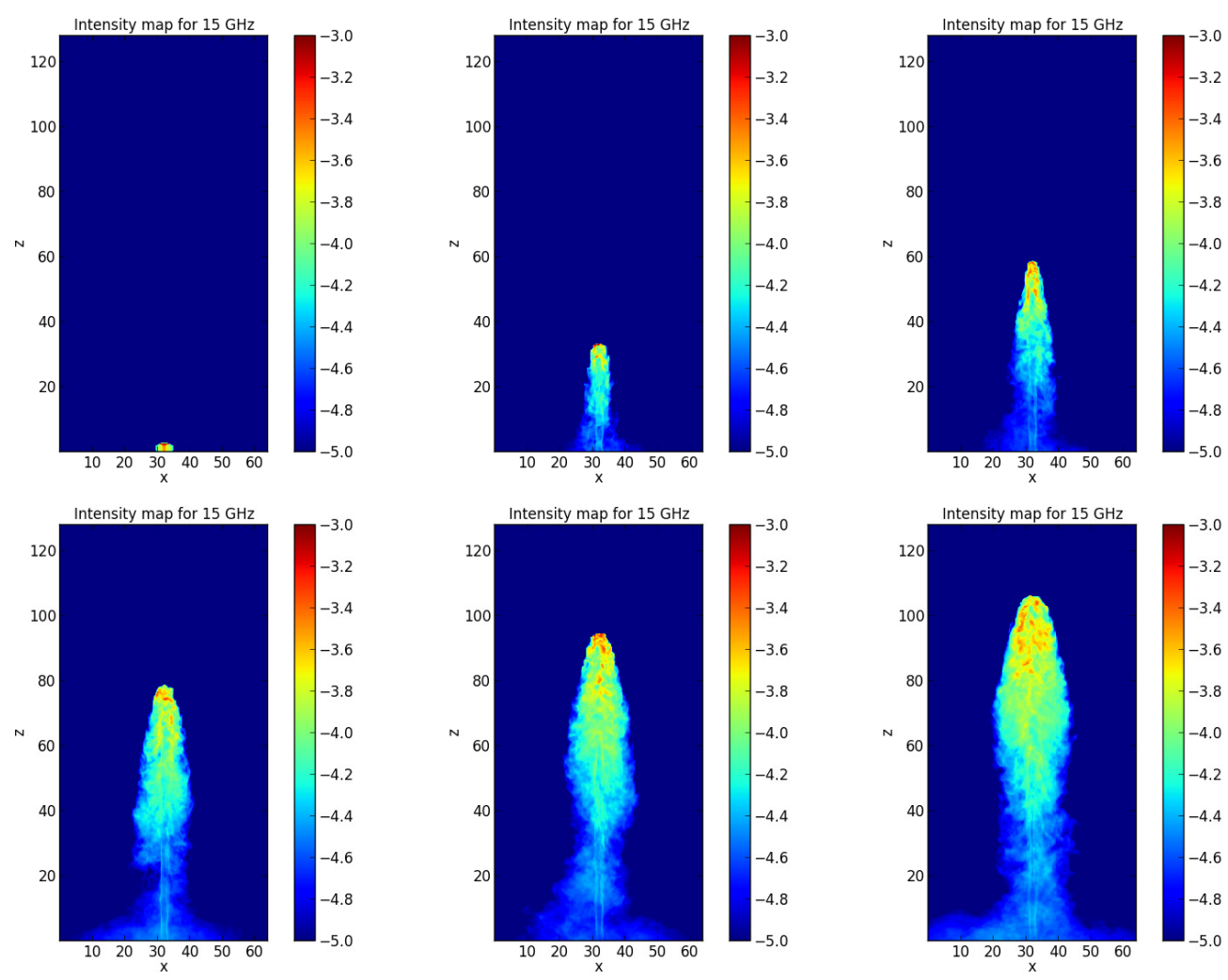

Figure 2: The emission observed from the jet simulation at six equal time steps. The scaling is logarithmic in arbitrary units to highlight the emission regions.

During the initial phase of the jet launch, the jet remains highly symmetrical. The emission is concentrated at the working surface, but is also seen in regions of standing shocks which form in the central beam of the jet. As the working surface propagates through the simulation the distance between the standing shock increases, while the jet remains symmetric.

As the jet continues to propagate through the medium it becomes more turbulent and the jet (and emission) appears less symmetric. While the peak in the emission region remains at the working surface, due to turbulence it is no longer symmetric around the original direction of injection and shifts around. The emission region is also no longer centred at a single location but is more spread out around the jet. The jet fluctuates between periods of lower peak intensity, with multiple "hot-spots" and periods of higher peak intensities which are more focused at the top of the jet.

The growth in turbulence is clearly shown by the variation in the density of the jet. Figure 3 shows the proper density plotted along the central $z$-axis of the beam (along the initial injection direction) for the same time steps as shown in figure 2 . The shock at the working surface, formed by the jet propagating into the ambient medium, shows the progress of the jet through the simulation. The density plots are taken at equal time steps (arbitrary time units) and shows how the propagation speed decreases as the jet moves through the medium, despite a continuous injection. Behind the working surface the standing shocks in the beam, formed due to the pressure difference between the jet beam and ambient medium, are seen as smooth oscillations in the density. The distance between 

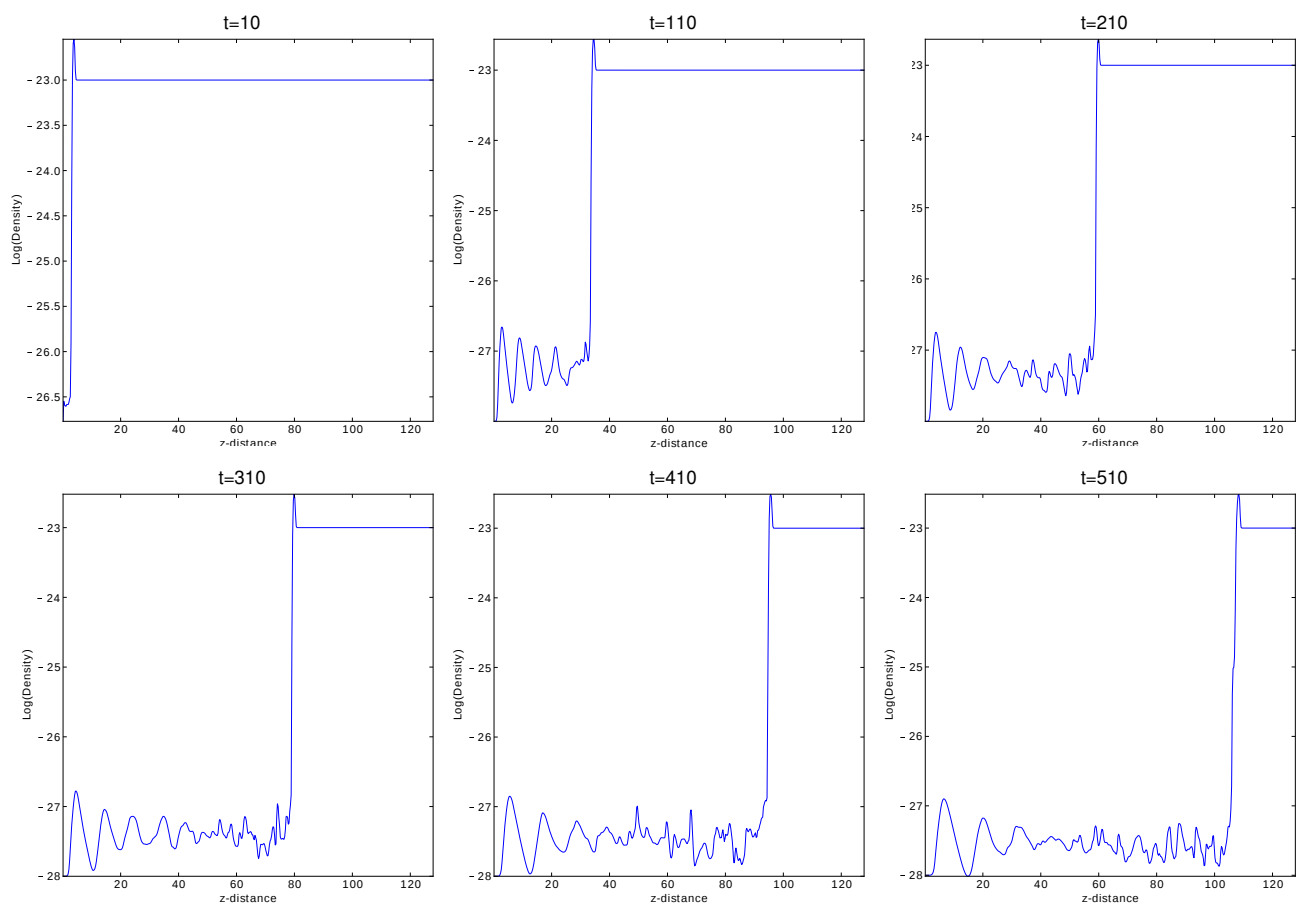

Figure 3: A one dimensional plot of the proper mass density (in arbitrary units) through the central beam of the jet (along the initial injection direction).

the standing shocks increases the further the jet travels and the density shows more turbulence, as is reflected in the emission maps (Fig. 2).

\section{Discussion and conclusions}

Here we have created an ideal relativistic jet simulation and used a post-processing emission code to produce intensity maps for synchrotron emission. The simulations show that even for this simplified case, of no magnetic field and constant injection the simulation produces variable emission with and changing jet morphology. The morphology of the jet is comparable to an FR II type jet, with the bulk of the emission concentrated at the end of the jet (see [14] for a discussion of forming FR I jets).

The variability observed from AGN is often modelled as the injection of "blobs" into the jet structure. We believe the injection of blob structures into our jet simulation will increase the complication of the simulations and produce more complicated structures.

The simulated emission has been calculated assuming a constant power-law distribution of electrons with an index of $p=1.8$ that is scaled with with the internal energy density. This first approximation is sufficient to highlight where the emission is most concentrated. This does, however, ignore the effects of cooling in the simulation. In future work we intent to expand the postprocessing code to model locations of particle acceleration, due to shocks, as well as the subsequent cooling of the electrons. In addition, here we have only shown the emission at a single frequency 
and we intend to expand the post-processing emission code to include inverse Compton scattering to simulated varying SEDs of AGN.

\section{Acknowledgments}

This work is based on the research supported by the National Research Foundation (NRF) of South Africa for the grant, No. 87919.

\section{References}

[1] F. Acero et al. Fermi Large Area Telescope Third Source Catalog, ApJS 218 (2015) 23 [doi: 10.1088/0067-0049/218/2/23]

[2] C.M. Urry, P. Padovani, Unified Schemes for Radio-Loud Active Galactic Nuclei, PASP 107 (1995) 803 [doi: 10.1086/133630]

[3] C.D. Dermer and G. Menon High Energy Radiation from Black Holes: Gamma Rays, Cosmic Rays, and Neutrinos, Princeton Univerisity Press 2009

[4] M. Böttcher, D.E. Harris and H. Krawczynski. (Eds.), Relativistic Jets from Active Galactic Nuclei, Wiley-VCH 2012

[5] B. Rani et al., Radio to gamma-ray variability study of blazar S5 0716+714, A\&A 552 (2013) A11 [doi: 10.1051/0004-6361/201321058]

[6] L. Fuhrmann et al., Detection of significant $\mathrm{cm}$ to sub-mm band radio and $\gamma$-ray correlated variability in Fermi bright blazars, MNRAS 441(2014) 1899 [doi: 10.1093/mnras/stu540]

[7] M. Błażejowski et al., A Multiwavelength View of the TeV Blazar Markarian 421: Correlated Variability, Flaring, and Spectral Evolution, ApJ 630 (2005) 130 [doi: 10.1086/431925]

[8] M. Böttcher et al., Leptonic and Hadronic Modeling of Fermi-detected Blazars, ApJ 768 (2013) 54 [doi: 10.1088/0004-637X/768/1/54]

[9] A. Mignone et al., PLUTO: a numerical code for computational astrophysics, ApJS 170 (2007) 228 [doi: 10.1086/513316]

[10] A. Mignone and G. Bodo, An HLLC Riemann solver for relativistic flows - I. Hydrodynamics, MNRAS 364 (2005) 126 [doi: 10.1111/j.1365-2966.2005.09546.x]

[11] E.F. Toro, Riemann solvers and numerical methods for fluid dynamics: a practical introduction, Springer, Berlin 2009

[12] M.-Á. Aloy et al., Three-dimensional Simulations of Relativistic Precessing Jets Probing the Structure of Superluminal Sources, ApJ 585 (2003) L109 [doi: 10.1086/374543]

[13] J.L. Gómez et al., Parsec-Scale Synchrotron Emission from Hydrodynamic Relativistic Jets in Active Galactic Nuclei, ApJ 449 (1995) L19 [doi: 10.1086/309623]

[14] S. Massaglia et al., Making Fanaroff-Riley I radio sources. Numerical Hydrodynamic 3D Simulations of Low Power Jets, A\&A 596 (2016) A12 [doi: 10.1051/0004-6361/201629375] 